This information is current as of April 25, 2023. 


\title{
Diagnostic Utility of Increased STIR Signal in the Posterior Atlanto-Occipital and Atlantoaxial Membrane Complex on MRI in Acute C1-C2 Fracture
}

\author{
(D) Y.-M. Chang, (D) Kim, (D) N. Peri, (DE. Papavassiliou, (DR. Rojas, and (DR.A. Bhadelia
}

\begin{abstract}
BACKGROUND AND PURPOSE: Acute C1-C2 fractures are difficult to detect on MR imaging due to a paucity of associated bone marrow edema. The purpose of this study was to determine the diagnostic utility of increased STIR signal in the posterior atlanto-occipital and atlantoaxial membrane complex (PAOAAM) in the detection of acute $\mathrm{Cl}-\mathrm{C} 2$ fractures on MR imaging.
\end{abstract}

MATERIALS AND METHODS: Eighty-seven patients with C1-C2 fractures, 87 with no fractures, and 87 with other cervical fractures with acute injury who had both CT and MR imaging within 24 hours were included. All MR images were reviewed by 2 neuroradiologists for the presence of increased STIR signal in the PAOAAM and interspinous ligaments at other cervical levels. Sensitivity and specificity of increased signal within the PAOAAM for the presence of a $\mathrm{Cl}-\mathrm{C} 2$ fracture were assessed.

RESULTS: Increased PAOAAM STIR signal was seen in 81/87 patients with C1-C2 fractures, 6/87 patients with no fractures, and 51/87 patients with other cervical fractures with $93.1 \%$ sensitivity versus those with no fractures, other cervical fractures, and all controls. Specificity was $93.1 \%$ versus those with no fractures, $41.4 \%$ versus those with other cervical fractures, and $67.2 \%$ versus all controls for the detection of acute $\mathrm{Cl}-\mathrm{C} 2$ fractures. Isolated increased PAOAAM STIR signal without increased signal in other cervical interspinous ligaments showed $89.7 \%$ sensitivity versus all controls. Specificity was $95.3 \%$ versus those with no fractures, $83.7 \%$ versus those with other cervical fractures, and $91.4 \%$ versus all controls.

CONCLUSIONS: Increased PAOAAM signal on STIR is a highly sensitive indicator of an acute $\mathrm{Cl}-\mathrm{C} 2$ fracture on MR imaging. Furthermore, increased PAOAAM STIR signal as an isolated finding is highly specific for the presence of a C1-C2 fracture, making it a useful sign on MR imaging when $\mathrm{CT}$ is either unavailable or the findings are equivocal.

ABBREVIATIONS: IDEAL = iterative decomposition of water and fat with echo asymmetric and least squares estimation; $\mathrm{NF}=$ no fracture; OF = other cervical fracture; PAOAAM = posterior atlanto-occipital and atlantoaxial membrane complex

n

njuries at the craniocervical junction occur in approximately $30 \%$ of patients presenting with blunt cervical spine trauma resulting in osseous and/or ligamentous injury. ${ }^{1,2}$ Given the high potential for neurologic morbidity associated with these injuries, accurate and timely assessment is critical for improved patient outcomes. ${ }^{3,4}$ Due to its high sensitivity for detecting acute fractures or dislocations by virtue of its high spatial resolution, ability to obtain multiplanar reformations, and speed, multidetector CT is the established initial imaging technique in patients suspected of having craniocervical and other cervical spine injuries. ${ }^{5-7} \mathrm{Al}-$ though MR imaging has superior ability for the evaluation of soft-

Received October 30, 2016; accepted after revision May 4, 2017.

From the Departments of Radiology (Y.-M.C., G.K., N.P., R.R., R.A.B.) and Neurosurgery (E.P.), Beth Israel Deaconess Medical Center, Boston, Massachusetts.

Please address correspondence to Yu-Ming Chang, MD, PhD, Department of Radiology, Beth Israel Deaconess Medical Center, WCB90, 330 Brookline Ave, Boston, MA 02115; e-mail: ychang2@bidmc.harvard.edu

http://dx.doi.org/10.3174/ajnr.A5284 tissue and spinal cord injuries and the determination of fracture acuity, its use is generally limited to cases with evidence of severe injuries on CT, abnormal neurologic findings, or equivocal CT findings. This secondary role of MR imaging in screening cervical spine injuries is driven not only by its higher cost, lower speed, and decreased availability compared with CT, but also by its decreased sensitivity for craniocervical junction and posterior element fractures. ${ }^{8,9}$

Recent work has shown that type II odontoid fractures in older patients may not exhibit STIR hyperintense marrow signal at the fracture site, limiting the utility of MR imaging in the evaluation of the presence and acuity of a fracture. ${ }^{10,11}$ Nevertheless, because of the potential for concomitant ligamentous and neurologic injuries associated with craniocervical fractures, MR imaging is frequently used as a complementary technique to CT at many tertiary care centers. ${ }^{12,13}$ In some of these instances, the initial CT or report obtained at an outside hospital may not be available for review by the radiologist inter- 
preting follow-up MR imaging in a timely fashion, or CT may have equivocal findings, creating a perfect scenario for missing $\mathrm{C} 1-\mathrm{C} 2$ fractures on MR imaging, with the potential for medicolegal implications.

We have observed increased signal on STIR images in the region of the posterior atlanto-occipital and atlantoaxial membranes, considered in the current study as a single complex (PAOAAM), in patients with acute C1-C2 fractures. However, this finding is typically not seen in patients presenting with trauma without cervical fractures, leading us to hypothesize that this may be a useful diagnostic indicator of acute C1-C2 fracture on MR imaging. Based on this hypothesis, the purpose of the study was to determine the diagnostic utility of increased PAOAAM STIR signal in the detection of acute C1-C2 fractures, the presence of which may prompt the reader to repeat or reinterpret CT cervical spine studies with equivocal findings.

\section{MATERIALS AND METHODS \\ Patients and Control Groups}

Following institutional review board approval for this Health Insurance Portability and Accountability Act-compliant study, a retrospective review from 2008 to 2015 of our institutional imaging data base was performed for patients with acute isolated C1-C2 fractures who had both CT and MR imaging of the cervical spine within 24 hours of each other. Per review of the medical records, the CT examinations were performed within 24 hours of trauma. CT performed at an outside hospital before transfer was included if it had been performed within 24 hours. All MR images were obtained at our institution. The presence of acute C1-C2 fractures was determined by the CT report and history/clinical examination documented in the medical records. One hundred two patients with $\mathrm{C} 1-\mathrm{C} 2$ fractures were identified. Nine fractures were determined to be chronic on the basis of review of prior imaging and/or clinical history and were excluded from analysis. Six additional patients were excluded due to the absence of diagnostic STIR images, leaving 87 patients.

Two control groups with CT scans obtained within 24 hours of blunt cervical spine trauma and MR images within 24 hours of the CT were selected consecutively by reviewing the imaging data base from 2013 to 2015 until each group included 87 patients. The controls consisted of a no fracture (NF) group and an other fracture (OF) group. The NF group included patients with a clinical history of blunt cervical spine trauma without reported fractures on CT. The OF group included all fractures isolated in the cervical spine (regardless of fracture morphology), excluding concomitant C1-C2 fractures.

\section{CT and MR Imaging}

Noncontrast CT of the cervical spine was performed on a $128-$ detector CT scanner (Discovery HD750; GE Healthcare, Milwaukee, Wisconsin). Images were acquired helically with a section thickness of $2.5 \mathrm{~mm}$, a pitch of 0.984 , a gantry rotation time of 1 second, at $120 \mathrm{kV}$ with a tube current of $340 \mathrm{~mA}$. All outside CT scans were also helically acquired and obtained on $64-$ or $128-$ detector CT scanners.

All MR imaging examinations were performed on one of two 1.5T MR imaging scanners with our standard departmental pro-

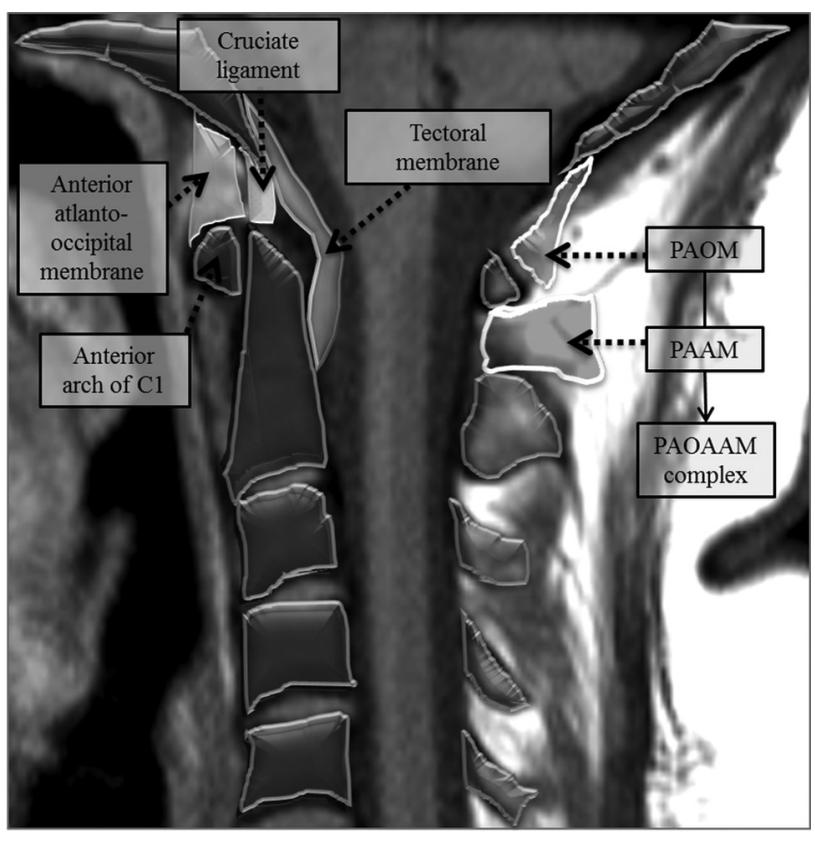

FIG 1. Overview of the anatomy at the craniocervical junction. The posterior atlanto-occipital membrane (PAOM) is a thickened band of the ligamentum flavum extending from the posterior arch of the atlas to the posterior occipital bone. The posterior atlanto axial membrane (PAAM) is a correlate extending from the posterior arch of the atlas to the posterior elements of $C 2$. The PAOM and PAAM are considered a single complex in this article (PAOAAM).

tocol of T1- and T2-weighted and STIR or STIR-equivalent iterative decomposition of water and fat with echo asymmetric and least squares estimation (IDEAL) sagittal water-selective images and axial T2-weighted and gradient recalled-echo images. On the Signa HDx scanner (GE Healthcare), STIR (2008-2012) imaging parameters were $\mathrm{TR} / \mathrm{TE}=3750 / 60 \mathrm{~ms}$ and $\mathrm{TI}=150 \mathrm{~ms}$, and IDEAL (2012-2015) imaging parameters were TR/TE $=4222 / 85$ $\mathrm{ms}$. For both sequences, the matrix was $320 \times 256$. For the Magnetom Espree (Siemens, Erlangen, Germany) scanner, the parameters were $\mathrm{TR} / \mathrm{TE}=5570 / 64 \mathrm{~ms}$, $\mathrm{TI}=150 \mathrm{~ms}$, and matrix $=$ $320 \times 224$. For both GE Healthcare and Siemens scanners, the FOV was $220 \mathrm{~mm}$ and the section thickness was $3 \mathrm{~mm}$.

\section{Image Analysis}

All CT scans obtained in our institution were interpreted by a boardcertified radiologist (emergency department radiologist or neuroradiologist). The subspecialty training of outside institution interpreters was not known. The CT studies were not independently re-reviewed by the investigators. For simplicity, STIR and IDEAL images will be collectively referred to as STIR images. STIR images from MR images were independently reviewed by 2 board-certified, fellowship-trained neuroradiologists (with 25 and 2 years of postfellowship experience), blinded to the CT reports and images, for the presence of increased signal on STIR images in the region of the PAOAAM. The increased STIR signal in the PAOAAM was defined as increased signal in one or both of the posterior atlanto-occipital and the posterior atlantoaxial membranes, which are considered in the current study as a single complex (PAOAAM) (Fig 1). STIR images were also used to assess the presence of increased signal in interspinous ligaments at other cervical levels (Figs 1-4). 

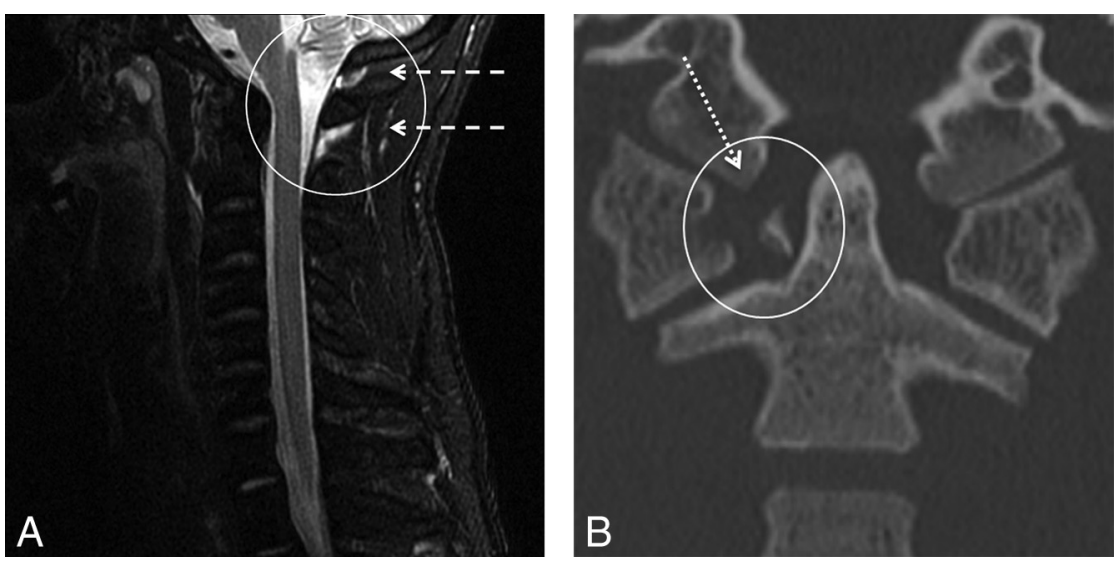

FIG 2. Isolated increased PAOAAM signal. A, STIR midline sagittal image of a 26-year-old man, presenting after assault. There is an isolated increased STIR signal (circle and dashed arrows) at the PAOAAM and none at the interspinous ligaments. B, CT demonstrates the anterior and posterior arches of $\mathrm{Cl}$ fractures, with an avulsed transverse ligament (circle and dotted arrow) and lateral subluxation of the lateral masses.
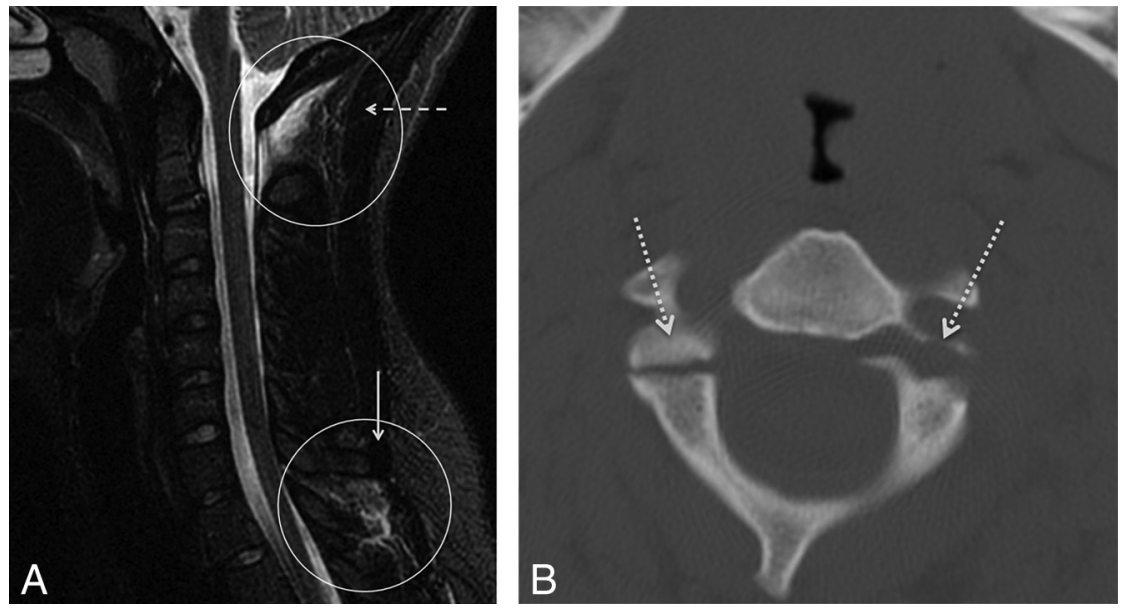

FIG 3. Increased PAOAAM signal. A, STIR midline sagittal image of a 17-year-old adolescent girl, presenting after a motor vehicle collision. There is an increased STIR signal at the PAOAAM (circle and dashed arrow) and at the interspinous ligaments (circle and solid arrow). B, CT demonstrates comminuted fractures of the bilateral C2 pedicles (dotted arrows).

\section{Data Analysis}

Differences in age among C1-C2, NF, and OF groups were assessed by using 1-way ANOVA. Interobserver reliability for both increased PAOAAM and interspinous ligament STIR signal was measured with $\kappa$ coefficients. Disagreements were resolved by consensus agreement between the 2 interpreters, and the reconciled data were used for subsequent analyses. The frequency of increased STIR signal in the PAOAAM and the presence of increased STIR signal in the interspinous ligaments at other cervical levels were determined for all patients. The sensitivity and specificity of increased STIR signal in the PAOAAM, both isolated to the PAOAAM and in the presence of STIR hyperintensity at other cervical levels, were assessed for the C1-C2, NF + OF (all controls), NF, and OF groups. We also assessed the sensitivity and specificity of isolated increased STIR signal in the PAOAAM associated with $\mathrm{C} 1-\mathrm{C} 2$ fractures by comparing these groups. Subgroup analysis of $\mathrm{C} 1-\mathrm{C} 2$ fracture types separated into odontoid type II, odontoid type III, C2 pars interarticular (hangman), C2 lamina or transverse process, C2 comminuted vertebral body, iso- lated $\mathrm{C} 1$, and combined $\mathrm{C} 1$ and $\mathrm{C} 2$ fractures with presence of increased STIR signal in the PAOAAM was performed with the $\chi^{2}$ test. Additional analysis of patients with NF separated into midcervical (C3 through $\mathrm{C} 4)$, lower cervical (C5 through $\mathrm{C} 7$ ), and mixed (C3 through C4 and C5 through C7) level fractures with presence of increased STIR signal in the PAOAAM was also performed. Statistical significance was set at $P \leq .05$.

\section{RESULTS}

Demographic characteristics of patients with $\mathrm{C} 1-\mathrm{C} 2$ fractures, NF, and OF are shown in Table 1. There were no significant age differences among the $\mathrm{C} 1-\mathrm{C} 2$ fracture, NF, and OF groups $(P \leq .2$, Table 1). With terminology established by Landis and Koch, ${ }^{14}$ there was "almost perfect" interobserver agreement between readers in the detection of PAOAAM signal $(\kappa=0.91 ; 95 \%$ CI, $0.88-0.97$ ) and interspinous ligament signal at other cervical levels $(\kappa=0.93 ; 95 \%$ CI, 0.89-0.98).

The 87 C1-C2 fractures were the following: odontoid type II ( $n=27)$, odontoid type III ( $n=19)$, C2 pars interarticular (hangman) $(n=7)$, C2 lamina or transverse process fractures $(n=3), \mathrm{C} 2$ comminuted vertebral body $(n=8)$, isolated $\mathrm{C} 1(n=20)$, and combined C1 and C2 $(n=3)$. Twenty-five of 27 odontoid type II, 18/19 odontoid type III, 7/7 hangman, 3/3 C2 lamina or transverse process, 8/8 C2 comminuted vertebral body, 19/20 isolated $\mathrm{C} 1$, and 3/3 combined $\mathrm{C} 1$ and $\mathrm{C} 2$ fractures demonstrated increased PAOAAM STIR signal. No significant difference was found in the presence of PAOAAM signal among the C1-C2 fracture groups $\left(\chi^{2}=1.52\right.$, $P=.96)$.

Fractures at other cervical levels included 16 mid- (C3 through C4), 59 lower- (C5 through C7), and 12 mixed-level fractures. Nine of 16 mid-, 33/59 lower-, and 9/12 mixed-level fractures were associated with increased PAOAAM STIR signal. No significant difference in the presence of PAOAAM signal among fractures involving mid-versus-lower cervical spine was noted $\left(\chi^{2}=\right.$ $1.54, P=.46)$.

Two patterns of increased STIR signal were observed in patients with acute C1-C2 fractures: 1) increased STIR signal in the PAOAAM with increased STIR signal in ligaments at other cervical levels (Fig 3), and 2) isolated increased STIR signal localized to the PAOAAM without increased signal in ligaments at other cervical levels (Fig 2).

Increased STIR signal in the PAOAAM was seen in $81 / 87$ 

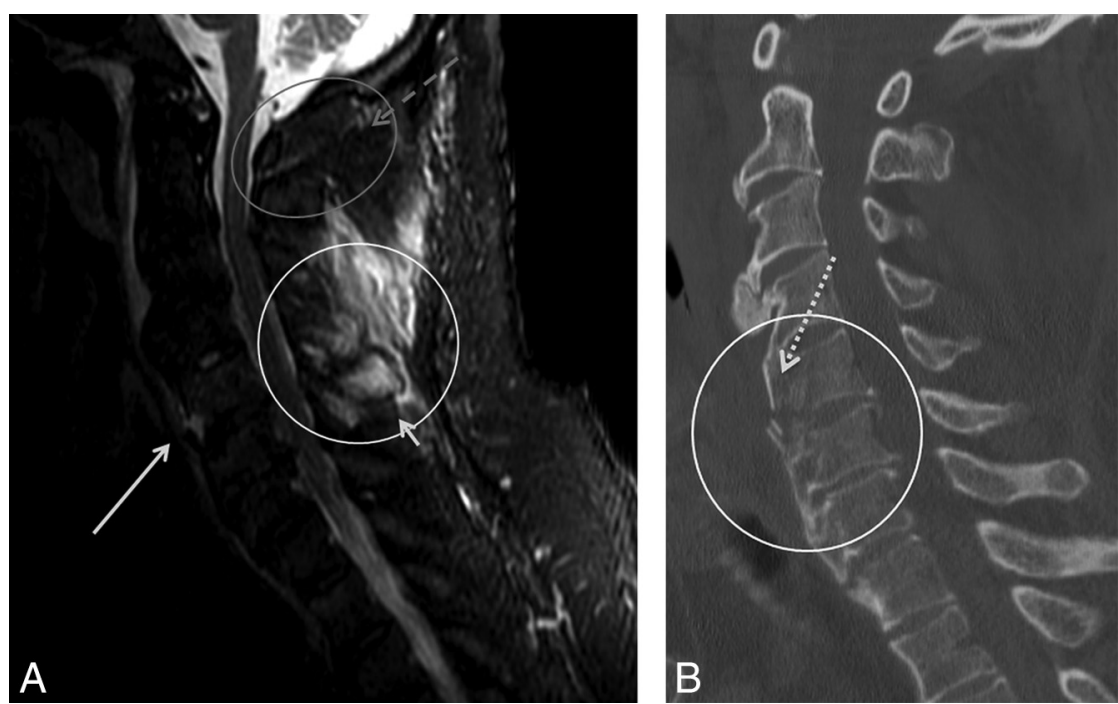

FIG 4. Other interspinous ligament signal. A, STIR midline sagittal image of a 66-year-old man presenting after a fall secondary to intoxication. There is no STIR signal at the PAOAAM (gray circle and dashed arrow), but only at the interspinous ligaments (white circle and short solid arrow). There is an interruption at the anterior longitudinal ligament at C5-6 (long solid arrow). $B$, $\mathrm{CT}$ demonstrates a fracture of the ossified anterior longitudinal ligament at C5-6 (circle and dotted arrow).

Table 1: Patient characteristics: sex distribution

\begin{tabular}{lccccc}
\hline & Male & Female & $\begin{array}{c}\text { Age } \\
\text { Range }(\mathbf{y r})\end{array}$ & $\begin{array}{c}\text { Mean } \\
\text { Age (yr) }\end{array}$ & SD \\
\hline C1-C2 fracture & 44 & 43 & $18-96$ & 66 & 21.4 \\
No fracture & 57 & 30 & $24-96$ & 62 & 17.5 \\
Other fracture & 50 & 37 & $21-102$ & 61 & 21.2 \\
\hline
\end{tabular}

(93.1\%) patients with C1-C2 fractures, 6/87 (6.9\%) with NFs, and $51 / 87(58.6 \%)$ with OFs. The sensitivity and specificity of increased STIR signal in the PAOAAM in detecting acute fractures at $\mathrm{C} 1-\mathrm{C} 2$ are shown in Table 2. Isolated increased STIR signal in the PAOAAM was seen in 52/87 (59.8\%) patients with C1-C2 fractures, $4 / 87$ (4.6\%) with NFs, and 7/87 (8.0\%) with OFs. The sensitivity and specificity of isolated increased STIR signal in the PAOAAM in detecting acute fractures at $\mathrm{C} 1-\mathrm{C} 2$ are shown in Table 3.

\section{DISCUSSION}

Our results show that increased STIR signal is observed in most patients with $\mathrm{C} 1-\mathrm{C} 2$ fractures and that this finding is a highly sensitive MR imaging sign of fractures in this area. We also observed that this finding has high specificity when compared with patients without cervical spine fracture (patients with NF). However, the specificity decreases when compared with patients with other cervical fractures (OF group) and all controls (NF + OF). This decreased specificity in relation to all controls and the OF group was due to the presence of increased STIR signal in the PAOAAM in many patients with other cervical spine fractures, suggesting that this structure is frequently affected in any type of cervical spine fracture. We also observed that when increased PAOAAM STIR signal is an isolated finding in patients with cervical spine injury and there is no associated increased signal in ligaments at other cervical levels, this finding has high specificity in indicating the presence of a C1-C2 fracture. On the basis of our observations, we believe that increased STIR signal in the PAOAAM should make a radiologist suspicious of the presence of a cervical spine fracture when interpreting a cervical spine MR imaging study. Furthermore, when the increased STIR signal in the PAOAAM is an isolated finding, a C1-C2 fracture should be suspected until proved otherwise by CT.

The STIR sequence on MR imaging is known to be very sensitive for the detection of a marrow edema pattern in subtle compression or micro-/insufficiency fractures, particularly of the thoracic and lumbar spines and appendicular skeleton, such as the pelvis and femur. ${ }^{15-18}$ Conversely, in the cervical spine, prior work has shown not only the superiority of CT in the detection of bony injuries, but also that STIR imaging is much less sensitive for the detection of acute fractures. Holmes et al, ${ }^{9}$ showed that of 66 patients with both CT and MR imaging examinations, the sensitivity for osseous fractures was $95 \%$ on CT versus $50 \%$ on MR imaging, with most of the missed lesions involving the lateral and posterior elements, with the caveat that the specific time interval between the CT and MR imaging studies was not reported by the authors.

Limitations of the STIR sequence were first demonstrated by Peri et al in 2009 in type II and III odontoid fractures in which STIR abnormalities were not seen in $22 \%$ of acute fractures and were limited to the fracture cleft in $11 / 18$ patients. ${ }^{10}$ In addition, a more recent study reported that the sensitivity of STIR signal for demonstrating acute type II odontoid fractures was only $82 \%$ in patients younger than 57 years of age and became significantly lower at $54 \%$ in patients older than 57 years of age. ${ }^{11}$ The mechanism underlying this difference in the sensitivity of MR imaging for the detection of acute fractures in the cervical spine versus the thoracic and lumbar spine is unclear; however, Lensing et $\mathrm{al}^{11}$ postulated that this issue may be due to a combination of progressive decreased vascularity at the odontoid base and age-related osteopenia.

Our findings also suggest that increased PAOAAM signal associated with C1-C2 fractures is not related to fracture morphology. Of note, prior work in the thoracolumbar spine reports that interspinous ligamentous injuries associated with fracture are correlated with the degree of kyphotic angulation and similar measures rather than fracture type. ${ }^{19,20}$ It is likely that the lack of correlation between $\mathrm{C} 1-\mathrm{C} 2$ fracture types and PAOAAM signal is due to the understanding that most cervical fractures are associated with high energy trauma, secondary to extreme flexion, extension, shearing, and rotation forces. Therefore, we believe that the findings outlined on our study can be generalized to any $\mathrm{C} 1-\mathrm{C} 2$ cervical fracture. ${ }^{21}$ In further support, cervical trauma associated with whiplash injury, presumably resulting from lower energy trauma than that associ- 
Table 2: Diagnostic utility of increased stir signal ${ }^{a}$ at the PAOAAM in detecting the presence of an acute $\mathrm{C} 1-\mathrm{C} 2$ fracture

\begin{tabular}{lccc} 
C1-C2 $(\boldsymbol{n}=81 / 87)$ vs & C1-C2 $(\boldsymbol{n}=81 / 87)$ vs & C1-C2 $(\boldsymbol{n}=81 / 87)$ vs \\
& All Controls $(\boldsymbol{n}=57 / 174)$ & NF $(\boldsymbol{n}=6 / 87)$ & OF $(\boldsymbol{n}=51 / 87)$ \\
\hline Sensitivity $(95 \% \mathrm{Cl})$ & $93.1 \%(85.6 \%-97.4 \%)$ & $93.1 \%(85.6 \%-97.4 \%)$ & $93.1 \%(85.6 \%-97.4 \%)$ \\
Specificity (95\% Cl) & $67.2 \%(59.7 \%-74.2 \%)$ & $93.1 \%(85.6 \%-97.4 \%)$ & $41.4 \%(30.9 \%-52.5 \%)$ \\
\hline
\end{tabular}

Note:- $\mathrm{Cl}-\mathrm{C} 2$ indicates all patients with $\mathrm{Cl}-\mathrm{C} 2$ fractures on $\mathrm{CT}$, with or without PAOAAM or another posterior ligament signal; NF, patients with a history of trauma without fracture on $\mathrm{CT}$, with or without PAOAAM or another interspinous ligament signal; OF, patients with cervical spine fracture other than $\mathrm{Cl}-\mathrm{C} 2$ on $\mathrm{CT}$, with or without PAOAAM or another interspinous ligament signal; controls, no cervical fracture plus other cervical fracture with or without PAOAAM or another interspinous ligament signal.

${ }^{a}$ Increased STIR Signal in PAOAAM with or without increased signal in other cervical ligaments.

Table 3: Diagnostic utility of isolated increased STIR signal ${ }^{a}$ at the PAOAAM in detecting the presence of an acute $\mathrm{C} 1-\mathrm{C} 2$ fracture

\begin{tabular}{lccc}
\hline & C1-C2 $(n=52 / 87)$ vs & C1-C2 $(n=52 / 87)$ vs & C1-C2 $(n=52 / 87)$ vs \\
& All Controls $(n=11 / 174)$ & NF $(n=4 / 87)$ & OF $(n=7 / 87)$ \\
\hline Sensitivity $(95 \% \mathrm{CI})$ & $89.7 \%(78.8 \%-96.1 \%)$ & $89.7 \%(78.8 \%-96.1 \%)$ & $89.7 \%(78.8 \%-96.1 \%)$ \\
Specificity $(95 \% \mathrm{CI})$ & $91.4 \%(85.1 \%-95.6 \%)$ & $95.3 \%(78.8 \%-96.1 \%)$ & $83.7 \%(69.3 \%-93.2 \%)$ \\
\hline
\end{tabular}

a Isolated increased STIR signal in the PAOAAM without increased signal in other posterior cervical ligaments.

ated with fractures, is not associated with PAOAAM injury compared with controls. ${ }^{22}$

The study has several limitations. First, we only looked at the STIR hyperintense signal of the PAOAAM and interspinous ligaments at other cervical levels and did not assess the marrow edema pattern or other soft-tissue signal in the current study. Although we specifically chose not to assess the marrow edema pattern in this study given the relatively lower sensitivity for odontoid and other craniocervical fractures on previous reports, such findings parenthetically noted may have resulted in some confirmation bias. Second, given that the OF and NF control groups were chosen consecutively among patients and not randomly, this method may have resulted in unforeseen selection bias. Third, because this report was focused on $\mathrm{C} 1-\mathrm{C} 2$ fractures, we did not analyze whether there were additional fracture types at the level of the craniocervical junction or skull base that may be associated with increased PAOAAM STIR signal. This omission could potentially result in lowered specificity of the findings for $\mathrm{C} 1-\mathrm{C} 2$ fractures. Further work elucidating this question will be required. We also did not independently review CT scans to confirm a diagnosis of cervical spine fracture, potentially resulting in over-/underestimation of the sensitivity and specificity of PAOAAM STIR signal for $\mathrm{C} 1-\mathrm{C} 2$ fractures. Finally, diagnosis of the acuity of the fracture was based on history/clinical findings, and we did not obtain a follow-up MR imaging to confirm whether and when PAOAAM increased signal subsequently resolved.

In our current study, we anecdotally noted 9 patients with a questionable history of prior $\mathrm{C} 2$ fracture whose cervical CT findings at the time of the new trauma were equivocal and later were determined to be chronic on the basis of prior imaging and/or clinical history and, therefore, were not included in the study. In these patients, the MR imaging performed within 24 hours of cervical CT did not demonstrate increased PAOAAM signal. Due to the small number of patients in this group, no definite conclusions could be drawn from this observation. Nevertheless, this finding suggests that increased signal at the PAOAAM is correlated with acute injury and is unlikely to be confounded by superimposed subacute-to-chronic injury. However, further study is required to address this issue. Furthermore, $6 / 87$ patients in the $\mathrm{C} 1-\mathrm{C} 2$ fracture group did not demonstrate as- sociated increased PAOAAM signal. The mechanism underlying this difference is uncertain and likely multifactorial, including mechanism of injury, cervical alignment, and bone density. For example, this subset of patients all demonstrated subjective decreased bone mineralization on CT examinations, though this was not confirmed by bone density examinations in the available medical records. Thus, it is possible that $\mathrm{C} 1-\mathrm{C} 2$ fractures in this false-negative group may occur with lower energy trauma and are less likely to result in ligamentous injury. Correlation with bone density in future studies would help elucidate these findings.

\section{CONCLUSIONS}

The presence of an increased PAOAAM signal on STIR images is a highly sensitive indicator of an acute $\mathrm{C} 1-\mathrm{C} 2$ fracture on MR imaging. As an isolated finding, increased PAOAAM signal is highly specific for the presence of a $\mathrm{C} 1-\mathrm{C} 2$ fracture, making it a diagnostically useful imaging sign for possible re-interpretation of reportedly negative/equivocal CT findings or repeat CT imaging if not available.

Disclosures: Rafael Rojas_UNRELATED: Consultancy: Guerbet, Comments: Guerbet MRI Advisory Board meeting, Chicago, July 2016.

\section{REFERENCES}

1. Riascos R, Bonfante E, Cotes C, et al. Imaging of atlanto-occipital and atlantoaxial traumatic injuries: what the radiologist needs to know. Radiographics 2015;35:2121-34 CrossRef Medline

2. Bohlman HH. Acute fractures and dislocations of the cervical spine: an analysis of three hundred hospitalized patients and review of the literature. J Bone Joint Surg Am 1979;61:1119-42 CrossRef Medline

3. Mendenhall SK, Sivaganesan A, Mistry A, et al. Traumatic atlantooccipital dislocation: comprehensive assessment of mortality, neurologic improvement, and patient-reported outcomes at a level 1 trauma center over 15 years. Spine J 2015;15:2385-95 CrossRef Medline

4. Hall GC, Kinsman MJ, Nazar RG, et al. Atlanto-occipital dislocation. World J Orthop 2015;6:236-43 CrossRef Medline

5. Berne JD, Velmahos GC, El-Tawil Q, et al. Value of complete cervical helical computed tomographic scanning in identifying cervical spine injury in the unevaluable blunt trauma patient with multiple injuries: a prospective study. J Trauma 1999;47:896-902; discussion 902-03 CrossRef Medline

6. Acheson MB, Livingston RR, Richardson ML, et al. High-resolution CT scanning in the evaluation of cervical spine fractures: comparison with plain film examinations. AJR Am J Roentgenol 1987;148: 1179-85 CrossRef Medline

7. Barba CA, Taggert J, Morgan AS, et al. A new cervical spine clearance protocol using computed tomography. J Trauma 2001;51:652-56; discussion 656-57 CrossRef Medline

8. Crim JR, Moore K, Brodke D. Clearance of the cervical spine in multitrauma patients: the role of advanced imaging. Semin Ultrasound CT MR 2001;22:283-305 CrossRef Medline

9. Holmes JF, Mirvis SE, Panacek EA, et al; NEXUS Group. Variability in computed tomography and magnetic resonance imaging in pa- 
tients with cervical spine injuries. J Trauma 2002;53:524-29; discussion 530 CrossRef Medline

10. Peri N, Papavassiliou E, Rojas R, et al. MRI of acute odontoid fractures: is marrow edema helpful in diagnosing "ACUTE" nature of fracture and predicting healing? In: Proceedings of the Radiological Society of North America Scientific Assembly and Annual Meeting; Chicago, Illinois. November 29 to December 4, 2009

11. Lensing FD, Bisson EF, Wiggins RH 3rd, et al. Reliability of the STIR sequence for acute type II odontoid fractures. AJNR Am J Neuroradiol 2014;35:1642-46 CrossRef Medline

12. Adams JM, Cockburn MI, Difazio LT, et al. Spinal clearance in the difficult trauma patient: a role for screening MRI of the spine. Am Surg 2006;72:101-05 Medline

13. Chew BG, Swartz C, Quigley MR, et al. Cervical spine clearance in the traumatically injured patient: is multidetector CT scanning sufficient alone? Clinical article. J Neurosurg Spine 2013;19:576-81 CrossRef Medline

14. Landis JR, Koch GG. The measurement of observer agreement for categorical data. Biometrics 1977;33:159-74 CrossRef Medline

15. Pizones J, Izquierdo $E$, Alvarez $P$, et al. Impact of magnetic resonance imaging on decision making for thoracolumbar traumatic fracture diagnosis and treatment. Eur Spine J 2011;20(suppl 3):390-96 CrossRef Medline

16. Spiegl UJ, Beisse R, Hauck S, et al. Value of MRI imaging prior to a kyphoplasty for osteoporotic insufficiency fractures. Eur Spine J 2009;18:1287-92 CrossRef Medline

17. Winklhofer S, Thekkumthala-Sommer M, Schmidt D, et al. Magnetic resonance imaging frequently changes classification of acute traumatic thoracolumbar spine injuries. Skeletal Radiol 2013;42:779-86 CrossRef Medline

18. Cabarrus MC, Ambekar A, Lu Y, et al. MRI and CT of insufficiency fractures of the pelvis and the proximal femur. AJR Am J Roentgenol 2008;191:995-1001 CrossRef Medline

19. Chen JX, Goswami A, Xu DL, et al. The radiologic assessment of posterior ligamentous complex injury in patients with thoracolumbar fracture. Eur Spine J 2017;26:1454-62 CrossRef Medline

20. Hiyama A, Watanabe M, Katoh $\mathrm{H}$, et al. Relationships between posterior ligamentous complex injury and radiographic parameters in patients with thoracolumbar burst fractures. Injury 2015;46:392-98 CrossRef Medline

21. Pratt $\mathrm{H}$, Davies E, King L. Traumatic injuries of the $\mathrm{C} 1 / \mathrm{C} 2$ complex: computed tomographic imaging appearances. Curr Probl Diagn Radiol 2008;37:26-38 CrossRef Medline

22. Dullerud R, Gjertsen O, Server A. Magnetic resonance imaging of ligaments and membranes in the craniocervical junction in whiplash-associated injury and in healthy control subjects. Acta Radiol 2010;51:207-12 CrossRef 